Original

\title{
Diagnóstico de factores de riesgo relacionados con la accidentabilidad de mano en trabajadores de una empresa refresquera
}

\author{
Diagnostic of risk factor related to the accidentes of hand in workers of solf-drinks \\ industry
}

\author{
Gabriela Betzabé Pérez-Manriquez', Mónica Sánchez-Aguilar1，Guadalupe González Díaz², Eduardo Oliva \\ López' ' Ignacio Enrique Peón Escalante'
}

1. Instituto Politécnico Nacional. Escuela Superior de Ingeniería, Mecánica y Eléctrica-Zacatenco. México, Distrito Federal.

2. Instituto Politécnico Nacional. Centro de Investigación e Innovación Tecnológica- Azcapotzalco. México, Distrito Federal.

Recibido: 31-01-12

Aceptado: 14-021-12

\section{Correspondencia}

Gabriela Betzabé Pérez Manriquez

Bosques de Mongolia, número 89

Colonia Bosques de Aragón

57170 Ciudad Netzahualcóyotl, Estado de México

Tfno: (0052) 5557664365

E-mail: dragaby2707@yahoo.com.mx

Resumen

Con el propósito de que se profundice en el reconocimiento de factores de riesgos derivados de este tipo de empresas y de estas actividades específicamente, para que se prevengan y minimicen los efectos a la salud de forma integral al interior de las empresas, el presente trabajo pretende determinar los factores de riesgo potencialmente relacionados con los accidentes de mano.

Objetivos: Determinar los factores de riesgo relacionados con los accidentes en mano para identificar que permitan disminuir la posibilidad de accidentes de mano mediante la modificación, eliminación y/o control de los factores presentes.

Material y métodos: Estudio observacional, descriptivo y longitudinal llevado a cabo en la población trabajadora del área de ventas, compuesta por 206 ayudantes y 81 agentes, de una Cooperativa productora Bebidas frutales de la Ciudad de México, en el periodo de 2007-2009. Se empleo el Método de Freeman Modificado para el Diagnóstico Situacional.

Resultados: Los factores de riesgo relacionados con accidentes de mano identificados y jerarquizados son: en primer lugar los psicosociales derivados de Organización Laboral (ritmo acelerado de la actividad) y la Condición Insegura (traslado del personal en la defensa trasera del camión); en segundo lugar se encontraron los Ergonómicos (movimientos repetitivos, manejo manual de cargas), junto con el Acto Inseguro (omisión del uso del equipo de protección personal), y la Condición Insegura (tipo de unidad, puerta y material transportado; y falta de capacitación); finalmente en tercer lugar los psicosociales de Organización Laboral (jornada indefinida mínima de 8 horas máxima de 14) y Condición Insegura (objetos punzocortantes en cajas).

Conclusiones: Los accidentes de mano constituyen un problema de salud pública importante a nivel nacional e internacional. Es de suma importancia identificar los factores de riesgo que se relacionan con su génesis como son: los factores psicosociales, condiciones inseguras, factores ergonómicos y actos inseguros. Por lo que se considera que estos, se deben buscar intencionadamente en las empresas dedicadas a este rubro de actividades, y evidenciarlos cuando se pretendan disminuir este tipo de accidentes y generar 
medidas preventivas y correctivas para mejorar la calidad de vida del trabajador y el rendimiento del mismo en sus labores.

Med Segur Trab (Internet) 2012; 58 (226) 13-26

Palabras clave: factores de riesgo, accidentes de mano.

Abstract

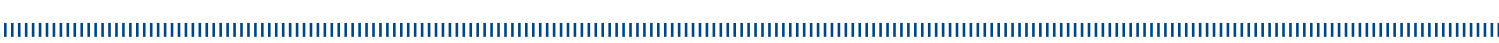

With the purpose of deepen on the recognize of risk factors from this kind of business and from this specific activities, to prevent and minimize the health effects integrally at the industries, the present work pretend to determine the risk factors potentially related with hand accidents.

Objective: Determine the risk factors related to the accidents of hand to identify and establish the risks allowing decrease the possibility of accidents in hand through the modification, elimination and or control of present factors.

Material and methods: Observational, descriptive and longitudinal study develop in worker population of sales area, composed by 206 helpers and 81 agents, cooperative producers of soft drinks and carbonated beverages in México City, in a period between 2007-2009. Was employed the Freeman Modified Method for the Situational Diagnostic.

Results: The risk factor related to hand accidents determined by the application of Freeman Modified Method in sales area according to the organization: were first derived from the psychosocial labor organization (accelerated pace of activity) and the Insecure Condition (transfer of the personnel within the rear bumper of the truck); second were found ergonomics (repetitive movements and manual handling of loads), along with Insecure Act (omission of the use of personal protection equipment), and the Insecure Condition (kind of unity, door and material transported, and lack of training); finally, in third psychosocial of Labor Organization (indefinite at least 8 hours maximum of 14) and unsafe (sharp boxes).

Conclusions: The risk factors presents in the generation of hand accidents in sales area of a soft drink industry are: primarily psychosocial factors due to the rhythm of the activity; unsafe condition due to lack of safety regulation. In second place ergonomic factors and unsafe acts by omission of use of personal protective equipment and unsafe condition, specifically lack of training and use of unsafe resources provided to the worker. Third place, psychosocial factors due to over 8 hours and under 14 hours working day.

Mentioned risk factors should be diagnosed promptly and to evidence in companies engages in the production of fruit drinks and carbonated, which seek to reduce work accidents though preventive and corrective measures.

Med Segur Trab (Internet) 2012; 58 (226) 13-26

Key words: risk factors, hand accidents. 


\section{INTRODUCCIÓN}

Desde una visión médica el presente trabajo tiene el objetivo de determinar los factores de riesgo potencialmente relacionados con los accidentes de mano y que son procedidos de la actividad de los trabajadores del área de ventas de una empresa refresquera. Con el propósito de que se profundice en el reconocimiento de factores de riesgos derivados de este tipo de empresas y de estas actividades específicamente, para que se prevengan y minimicen los efectos a la salud de forma más integral al interior de las empresas.

En México, los Riesgos de Trabajo según el Título IX, Art. 473 de la Ley Federal del Trabajo, son los accidentes y enfermedades a que están expuestos los trabajadores en ejercicio o con motivo del trabajo. Estos Riesgos de Trabajo constituyen uno de los problemas contemporáneos más importantes para la salud de los trabajadores en todo el mundo. Las tasas de incidencia de este tipo de factores son significativamente elevadas en comparación con otros países ${ }^{1,2}$.

Dentro de las estadísticas de salud del Instituto Mexicano del Seguro Social (IMSS), los riesgos de trabajo, específicamente los accidentes, que para fines de esta investigación se consideran los ocurridos dentro de la jornada laboral y con motivo del ejercicio de las actividades implicadas en el proceso de trabajo del personal de ventas, han ocupado un lugar relevante durante los últimos años (ver tabla I), convirtiéndose en un serio problema de Salud Pública para México.

Este problema debe ser valorado y desde luego, atendido en forma integral, tanto por las implicaciones monetarias y médico-psicológicas que repercuten en la economía y en la salud de los trabajadores, de sus familias y de la sociedad en general, como por las repercusiones e implicaciones patronales y la productividad nacional erogada.

Se estima que en los países desarrollados el 4\% del Producto Interno Bruto es perdido debido a los riesgos de trabajo y esto puede acercase al $10 \%$ en países en vías de desarrollo ${ }^{1}$.

En la tabla I se observa que durante el periodo de 2000-2009 se han afiliado un promedio de 808, 000 empresas, las cuales han reportaron a lo largo de esta década un promedio de 418, 300 casos de riesgos de trabajo. De los cuales 331,700 casos corresponden únicamente a accidentes de trabajo y sólo 5,300 casos a enfermedades ocupacionales, es decir, el $79.2 \%$ han sido accidentes y sólo el $1.2 \%$ son enfermedades de trabajo, el resto se trata de accidentes de trayecto ${ }^{3}$.

Tabla I. Estadísticas de Empresas y Trabajadores Afiliados, Riesgos de Trabajo e Incapacidades Permanentes por Tipo de Riesgo en el Período 2000-2009

\begin{tabular}{c|c|c|c|c|c|c|c|c|c|c}
\hline CONCEPTOS & 2000 & 2001 & 2002 & 2003 & 2004 & 2005 & 2006 & 2007 & 2008 & 2009 \\
\hline $\begin{array}{c}\text { EMPRESAS } \\
\text { CON SEGURO } \\
\text { DE RIESGOS } \\
\text { DE TRABAJO } \\
(1)\end{array}$ & 776020 & 800617 & 804389 & 804389 & 804389 & 802107 & 810181 & 823999 & 833072 & 825159 \\
\hline $\begin{array}{c}\text { TRABAJADORES } \\
\text { BAJO SEGURO } \\
\text { DE RIESGOS } \\
\text { DE TRABAJO } \\
(1)\end{array}$ & 12418761 & 12224231 & 12112405 & 12088468 & 12348259 & 12735856 & 13578346 & 14424178 & 14260309 & 13814544 \\
\hline $\begin{array}{c}\text { RIESGOS DE } \\
\text { TRABAJO }\end{array}$ & 454089 & 413748 & 387806 & 358784 & 360793 & 373239 & 387827 & 450102 & 506934 & 489787 \\
\hline $\begin{array}{c}\text { Accidentes de } \\
\text { trabajo }\end{array}$ & 356725 & 324150 & 302970 & 278525 & 282469 & 295594 & 309539 & 361244 & 411179 & 395024 \\
\hline $\begin{array}{c}\text { Enfermedades } \\
\text { de trabajo }\end{array}$ & 5557 & 5520 & 4511 & 7811 & 7418 & 7292 & 4715 & 2691 & 3681 & 4101 \\
\hline $\begin{array}{c}\text { Accidentes y } \\
\text { enfermedades } \\
\text { de trabajo }\end{array}$ & 362282 & 329670 & 307481 & 286336 & 289887 & 302886 & 314254 & 363935 & 414860 & 399125 \\
\hline
\end{tabular}




\begin{tabular}{c|c|c|c|c|c|c|c|c|c|c}
\hline CONCEPTOS & 2000 & 2001 & 2002 & 2003 & 2004 & 2005 & 2006 & 2007 & 2008 & 2009 \\
\hline $\begin{array}{c}\text { INCAPACIDADES } \\
\text { PERMANENTES }\end{array}$ & 22117 & 20006 & 20427 & 22964 & 21831 & 20693 & 19327 & 17642 & 18999 & 20477 \\
\hline $\begin{array}{c}\text { Accidentes de } \\
\text { trabajo }\end{array}$ & 14202 & 12433 & 13084 & 11700 & 11916 & 11578 & 12555 & 12094 & 13603 & 14530 \\
\hline $\begin{array}{c}\text { Enfermedades } \\
\text { de trabajo }\end{array}$ & 6763 & 6532 & 6220 & 10235 & 8837 & 8143 & 5585 & 4321 & 3884 & 4191 \\
\hline
\end{tabular}

(1) Estimado con base en el cuadro 16 Informe Mensual de Población Derechohabiente, enero - diciembre de cada año.

Fuente: Memorias Estadísticas IMSS, Coordinación de Medicina del Trabajo, 2000-2009

En 2009, se observó que el rango de edad con mayor incidencia de accidentes de trabajo se encuentra entre los 20 a 24 años de edad y que el sexo masculino ha ocupado el primer lugar en accidentes de trabajo, con promedio de 268,504 casos $(80.9 \%)$ en comparación con el sexo femenino, el cual ha presentado en promedio un total de 130, 950 casos $(39.4 \%)^{3}$. (Ver tabla II y III)

Tabla II. Riesgos de Trabajo, Accidentes y Enfermedades de Trabajo por Sexo, 2009

\begin{tabular}{c|c|c|c|c|c}
\hline \multicolumn{2}{c|}{$\begin{array}{c}\text { Riesgos de Trabajo } \\
\text { por Sexo 2009 }\end{array}$} & \multicolumn{2}{c|}{$\begin{array}{c}\text { Accidentes de Trabajo } \\
\text { por Sexo 2009 }\end{array}$} & \multicolumn{2}{c}{$\begin{array}{c}\text { Enfermedades de Trabajo } \\
\text { por Sexo 2009 }\end{array}$} \\
\hline $\begin{array}{c}\text { Total de Riesgos } \\
\text { de trabajo }\end{array}$ & 489787 & $\begin{array}{c}\text { Total de } \\
\text { Accidentes } \\
\text { de trabajo }\end{array}$ & 395024 & $\begin{array}{c}\text { Total de } \\
\text { Enfermedades } \\
\text { de trabajo }\end{array}$ & 4101 \\
\hline Hombres & 313763 & Hombres & 269942 & Hombres & 3237 \\
\hline Mujeres & 143491 & Mujeres & 96762 & Mujeres & 670 \\
\hline
\end{tabular}

Fuente: Memorias Estadísticas IMSS, Coordinación de Medicina del Trabajo, 2000-2009

Tabla III. Principales grupos etarios relacionados con Accidentes y Enfermedades de Trabajo, 2009

\begin{tabular}{|c|c|c|c|c|}
\hline Orden Jerárquico & $\begin{array}{c}\text { Grupos de edades con más } \\
\text { Accidentes y Enfermedades } \\
\text { de Trabajo }\end{array}$ & $\begin{array}{c}\text { Accidentes de } \\
\text { Trabajo }\end{array}$ & $\begin{array}{c}\text { Enfermedades } \\
\text { de Trabajo }\end{array}$ & $\begin{array}{c}\text { Total de } \\
\text { Casos }\end{array}$ \\
\hline $1 .^{o}$ & 25-29 años & 71324 & 227 & 89813 \\
\hline $2 .^{\circ}$ & 20-24 años & 69656 & 159 & 84869 \\
\hline $3 .^{\circ}$ & 15-19 años & 18536 & 33 & 21305 \\
\hline
\end{tabular}

Fuente: Memorias Estadísticas IMSS, Coordinación de Medicina del Trabajo, 2000-2009

En cuanto a la región del cuerpo más afectada a lo largo de esta década ha sido la mano y muñeca la de mayor incidencia de accidentes de trabajo como se muestra más adelante en la gráfica $1^{3}$.

\section{CONTEXTO}

\section{Factores de riesgo}

Los factores de riesgo para accidentes de trabajo, para efecto de esta investigación, son aquellos que aumentan o favorecen la probabilidad de ocurrencia de un accidente. Se clasifican en los siguientes grupos:

- Factores o condiciones de seguridad.

- Factores de origen físico, químico, biológico, ergonómico o condiciones medioambientales.

- Factores derivados de las características del trabajo.

- Factores derivados de la organización del trabajo. 
A lo largo del tiempo y después de cada riesgo materializado, se busca establecer cada uno de los factores de riesgo presentes en el lugar de trabajo que dieron o pueden dar lugar al accidente. ${ }^{[4]}$ Es por ello, que tanto México como otros países han tratado de identificar los factores que se han visto relacionados con la génesis de accidentes de mano en distintos giros de la industria alimentaria, como se muestra más adelante, y entre los que se encuentran:

1. Distracción.

2. Mal uso o inadecuada selección del equipo de protección personal.

3. Falta de capacitación y entrenamiento.

4. Jornadas prolongadas.

5. Ejecución de métodos de trabajo diferentes al estándar.

6. Ejecución de actividades nuevas.

7. Enfermedad previa.

8. Velocidad en la ejecución de la tarea.

9. Poca experiencia.

10. Exceso de confianza.

11. Manejo manual de cargas.

12. Movimientos repetitivos, entre otros.

Cabe aclarar que dada la escasa investigación nacional e internacional reportada acerca de accidentes laborales de mano en el ramo refresquero, la información que se muestra se obtiene de reportes de investigaciones de otras empresas productoras o procesadoras de alimentos en general, así como de empresas manufactureras de botellas de vidrio donde la manipulación de las mismas es el riesgo al que se exponen los trabajadores de ventas de las empresas refresqueras durante el desarrollo de su actividad laboral.

Además de los factores enlistados anteriormente, en algunos estudios realizados en México sobre factores de riesgo, tipo de gravedad y secuelas permanentes en las lesiones de mano de origen profesional, durante los años 2003-2004, se encontraron: "el día lunes", como factor de riesgo relacionado con accidentes que dejan lesiones graves y permanentes en mano por motivo laboral; la "falta de capacitación", como condicionante de este tipo de accidentes; "sexo masculino" el más afectado; y "antecedentes de toxicomanía". ${ }^{[5]}$ Esto permite ver algunos de los factores de riesgo que se deben buscar intencionadamente al momento de realizar un Diagnóstico Situacional y al buscar disminuir la accidentabilidad de los trabajadores en la empresa.

A nivel internacional, como se mencionó, también existe la misma preocupación por establecer los factores de riesgo relacionados con la generación de accidentes de trabajo, y se han encontrado algunos otros factores de riesgo además de los observados en la lista previa, o bien, los confirman.

Por ejemplo, en India, durante un estudio (tipo encuesta) a los trabajadores del proceso de trituración de caña, proceso en el cual las manos son la región del cuerpo mayormente expuesta y la más afectada, se observó que el 63\% de los casos de accidentes se debieron a "distracción", el 84\% de los trabajadores concluyeron que la "atención y el cuidado en el trabajo" pueden reducir los riesgos. El 16\% expresó que el "uso de maquinaria" más segura disminuiría el riesgo. El 40\% de los empleados declararon que sería de gran utilidad el "uso de guantes", mientras que el $19 \%$ pensaron que sería un obstáculo; sin embargo, ninguno había intentado emplearlos, y sólo el $28 \%$ se encontró en la disposición de usarlos. Otros factores observados fueron el "bajo índice de alfabetización" y "recursos económicos bajos". Pese a que en otros estudios se ha demostrado que la "jornada prolongada" aumenta el descuido y por ende los accidentes, en este caso no se consideró un factor de riesgo, a pesar de que la jornada de estos 
trabajadores era de 12 horas. Se concluyó que la "capacitación y entrenamiento" podría reducir la morbilidad de lesiones ${ }^{6}$.

Hong Kong, también hace evidente su preocupación por establecer factores de riesgo relacionados con los accidentes de mano. Durante el año 2007, se realizó un estudio de factores de riesgo transitorios para lesiones traumáticas agudas de mano, en donde se encontraron siete factores de riesgo relacionados: 1) mal uso del equipo de protección personal y de materiales, 2) uso de un método de trabajo diferente al estipulado, 3) ejecución de una actividad nueva, 4) trabajo extra, 5) sensación de enfermedad, 6) distracción, y 7) apresuramiento. Como se puede observar, estos factores son modificables, por lo que el estudio recomienda aumentar la concientización sobre estos factores entre los trabajadores y patrones para encaminar los esfuerzos a evitar la exposición mediante medidas de ingeniería y administrativas complementadas con la educación en seguridad, capacitación y adiestramiento ${ }^{7}$.

Con respecto a los factores ergonómicos, la manipulación manual de cargas es responsable, en muchos casos, de la aparición de fatiga física (en este caso localizada en la mano), o bien de lesiones, que se pueden producir de una forma inmediata o por la acumulación de pequeños traumatismos, aparentemente sin importancia pero que a largo plazo se hacen tangibles en la génesis de un accidente. Estas lesiones pueden presentarse tanto en los trabajadores que manipulan cargas regularmente $\mathrm{u}$ ocasional ${ }^{8,9}$.

Se ha visto que el uso de pequeñas herramientas y el trabajo manual de manera frecuente, expone al trabajador a lesiones en su mayoría menores, que a largo plazo se pueden volver graves, de no ser atendido a tiempo el factor generador ${ }^{10}$.

Al respecto, se observa que cuando al trabajo manual y repetitivo se le añade el factor carga, las posibilidades de lesiones o accidentes aumentan considerablemente. Esto debido a que se incrementa el esfuerzo muscular como consecuencia de las cargas, disminuyendo así la circulación sanguínea muscular, apareciendo más rápidamente la fatiga y con ello la posibilidad de un accidente. El conjunto de problemas asociados a las tareas con carga física supone unas pérdidas de unos 600 millones de jornadas al año ${ }^{11}$.

En estudios realizados en India, en una empresa procesadora de pescado y, en Singapur en trabajadores de puestos que implicaban el manejo manual de cargas en diversas industrias, se concluyó que aquellos factores de riesgo ergonómicos, como el "manejo manual de cargas" y los "movimientos repetitivos", son una condicionante de accidentes en miembros superiores, dejando lesiones como cortaduras, fracturas, contusiones $^{12,8}$.

Con esto se concluye que aunque desafortunadamente las investigaciones sobre accidentes de mano en la industria alimentaria es escasa, se han hecho grandes esfuerzos para establecer los factores de riesgo relacionados con este tipo de accidentes, lo que puede ayudar a tener bien establecidos los factores a abordar al momento de tener una estadística elevada de accidente de mano en las empresas y así ser resueltos más rápidamente con las medidas integrales pertinentes.

Como se mencionó con anterioridad, dada la relación que guarda la actividad que desempeñan los trabajadores de ventas de una empresa refresquera con la manipulación de vidrio, existe una cierta igualdad de riesgos que comparten los trabajadores estudiados. Durante una investigación realizada en una planta manufacturera de botellas de vidrio en India en 1998, se observó que los factores de riesgo más significativos fueron la "edad (<30 años)", y la "experiencia menor a 2 años"; los factores técnicos responsables de daño fueron dependientes de alteraciones en el sitio de trabajo, equipo de protección personal inadecuado, mal manejo de máquinas, y los factores humanos más presentes fueron la omisión del uso del equipo de protección personal, el exceso de confianza, y los errores de procedimiento al manipular las máquinas ${ }^{13}$.

También en la India, se observaron otros factores de riesgo que guardan relación con los accidentes como son: la característica de la política salarial (mayor sueldo por destajo) y la temporalidad del trabajo (trabajo temporal), estos a su vez favorecen otros 
factores de riesgo adicionales a los ya mencionados, tales como: "tabaquismo, alcoholismo, factores psicosociales, rotación de turnos o puestos, mayor velocidad del trabajo”. Además se encontró que la "condición de trabajo temporal" incrementa la severidad y frecuencia de dichos accidentes. Este estudio concluyó que el trabajo temporal tiene un riesgo más alto de accidentes, lo que podría deberse a la "experiencia menos eficaz", así como a la "carencia de seguridad" en el puesto ${ }^{14}$.

\section{Accidentes de trabajo}

Cuando cada riesgo presente en el lugar de trabajo se llega a materializar y deja de ser una simple probabilidad para convertirse en un accidente, para el trabajador o para los recursos de la organización, es cuando cobra importancia el estudio de ambos factores a nivel nacional y mundial ${ }^{4}$.

En México, en materia de accidentes de trabajo el IMSS, en sus estadísticas de los últimos 10 años, ha reportado un promedio de 418,300 riesgos de trabajo. De los cuales 331,700 casos corresponden únicamente a accidentes (79.2\%) y sólo 5,300 casos a enfermedades ocupacionales (1.2\%), el resto se trata de accidentes de trayecto. De igual manera, a lo largo de estos últimos 10 años, se ha observado que el rango de edad con mayor incidencia de accidentes se encuentra entre los 20 a 24 años de edad y que el sexo masculino ha ocupado el primer lugar, con un promedio de 268,504 casos (80.9\%) en comparación con el sexo femenino, el cual ha presentado un promedio total de 130,950 casos (39.4\%). En cuanto a las regiones del cuerpo más afectadas han sido la mano y muñeca en cuanto a incidencia de accidentes, como se muestra en la gráfica 1.

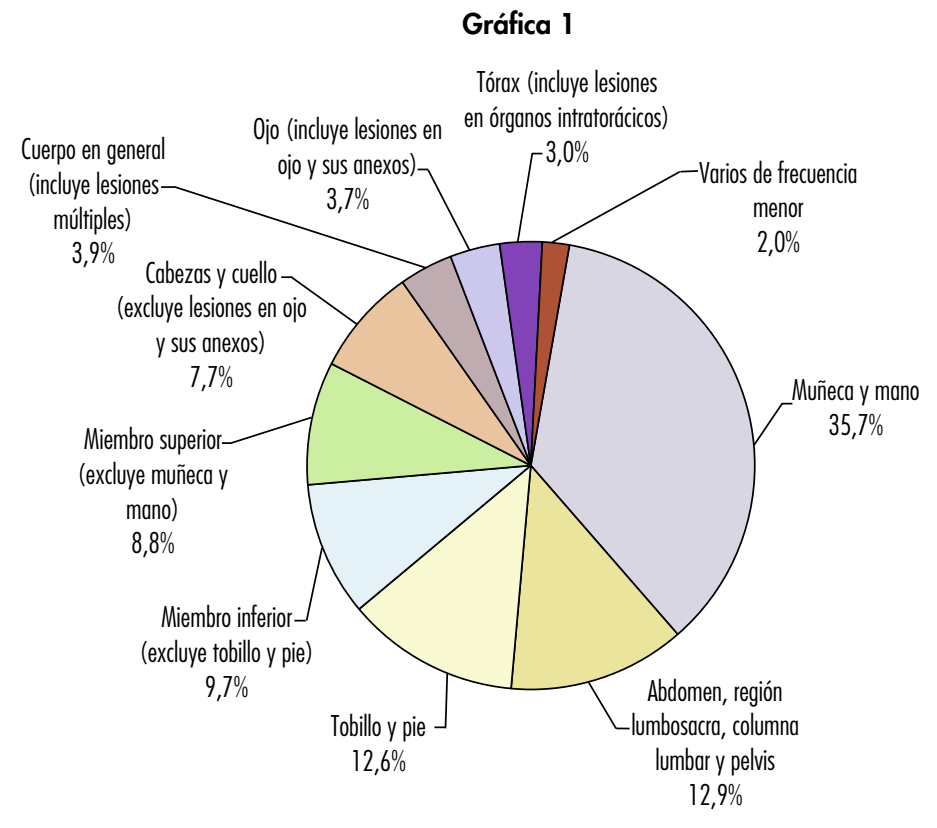

Fuente: Memorias Estadísticas IMSS, Coordinación de Medicina del Trabajo, 2001

Las consecuencias de los accidentes de mano, según han evidenciado la Facultad de Medicina de la Universidad Nacional Autónoma de México (UNAM) y el IMSS en los últimos 10 años son: fracturas, quemaduras y laceraciones ${ }^{15}$.

En el estado de Veracruz (estado que ha publicado información al respecto), en el año 2005, mediante una comparación de las características de la accidentabilidad entre trabajadores del IMSS y los de empresas afiliadas, se encontró que las lesiones más habituales a las que se enfrenta la mano dentro del medio laboral son: heridas, fracturas y luxaciones. Se vio que entre los "trabajadores IMSS" la accidentabilidad predominó en el sexo femenino, con edad entre 37 a 42 años, antigüedad en el puesto de 15 años, personal capacitado en más de la mitad de los casos, y que percibían un salario mínimo. 
En comparación, entre los "trabajadores de empresas afiliadas al instituto", la accidentabilidad predominó en el sexo masculino, con edad entre 27 a 36 años, antigüedad menor de un año, capacitados en más de la mitad de los casos, que percibían un salario mayor al mínimo, quienes presentaron diagnósticos de quemadura y fractura, esguince, heridas y amputación, en las áreas de la mano mayormente afectadas: dedos, mano y muñeca ${ }^{16}$.

Una de las industrias que más número de accidentes deja cada año es la de alimentos. Un estudio realizado en Cd. Victoria, Tamaulipas, en una productora de tortillas mostró que las lesiones graves en los dedos y manos se han incrementado; y que este tipo de lesiones generan amputaciones debido a que no se utiliza ningún implemento de seguridad, de tal manera que se sufre el accidente por distracción, exceso de confianza, falta de capacitación o bien, durante reparaciones: por ajuste de bandas y por uso de herramientas inadecuadas ${ }^{17}$.

Al igual que en México, a nivel mundial se busca establecer cuáles son las principales lesiones que afectan al personal cuando se presenta un accidente de trabajo. Según la Organización Internacional del Trabajo (OIT), aproximadamente 270 millones de accidentes están relacionados con el trabajo y hay aproximadamente, 2 millones de muertes cada año debido a la misma causa ${ }^{18}$.

Es importante mencionar que, la OIT considera al accidente de trabajo como la consecuencia de una cadena de factores en la que algo ha funcionado mal y no ha llegado a buen término. ${ }^{[14]}$ Se estima que 50 millones de trabajadores sufren algún tipo de accidente anualmente, lo cual se refleja en 160 mil accidentes cada día ${ }^{13}$.

A nivel internacional, en Estados Unidos de Norteamérica (E.U.A), también se ha visto que la mano es el principal sitio de accidentes durante el ejercicio laboral y el más atendido en unidades de emergencia hospitalaria y se estima que se presenta en 1,080,000 trabajadores anualmente, siendo las principales lesiones: laceraciones y cortaduras. El aumento perceptible del riesgo para las manos puede deberse al uso inadecuado de equipo de protección, a que este esté defectuoso, a la realización de tareas inusuales, periodos largos de trabajo; a la edad, el género, la experiencia en el puesto y/o el poco entrenamiento de seguridad. De igual forma se observó que el $60 \%$ de los accidentes fueron debidos a condiciones inseguras ${ }^{19}$.

En Italia, durante un estudio del total de lesiones y accidentes fatales en los diferentes sectores de la industria, que comprendió el periodo de 1951 a 1998, se encontró que la mayor incidencia de accidentes se daba en los rubros industriales: alimentos y agricultura, química, y metalurgia. Los principales accidentes reportados fueron: los aplastamientos o amputaciones, en primer lugar; en segundo, la colisión; en tercero, las lesiones autoprovocadas; en cuarto, las caídas del plano de bipedestación; en quinto, las caídas por distracción o adormecimiento; y en último lugar, las caídas de un nivel a otro. Dentro del rubro de otras, se encontraron las caídas en alguna máquina abierta, movimientos no coordinados y daños por objetos pesados ${ }^{20}$.

Según estadísticas españolas de accidentes con incapacidad permanente, se reporta que el $25 \%$ de estos accidentes fueron generados por el sobreesfuerzo secundario al trabajo manual de cargas, el $\mathbf{1 8 . 8 \%}$ fueron debidos a golpes con objetos y herramientas, o caídas de personas del mismo o diferente nivel $(9.8 \%$ y $8.3 \%$ de los accidentes, respectivamente). Aunque, en general, la gravedad de estos accidentes producidos por el manejo manual de cargas de manera repetitiva, es inferior a la de otros tipos de accidentes, las consecuencias que tienen en conjunto, son muy importantes para las industrias, como es el número de jornadas perdidas ${ }^{8,9}$. 


\section{DESCRIPCIÓN DEL TRABAJO DE VENTAS}

El trabajo de ventas que se aborda durante este estudio, se refiere al realizado por trabajadores dedicados al reparto y entrega del producto terminado para su venta a detalle (refiriéndose a la venta que se hace a los dueños de tiendas o establecimientos comerciales). Algunas de las características que presenta este tipo de empleo son: paga por trabajo a destajo, estatus temporal, vulnerabilidad.

En México, el IMSS reportó en sus estadísticas recientes (2007), que el puesto de vendedor es el segundo lugar en presentar accidentes de trabajo, con un total de 20,172 casos a nivel nacional; observándose que el sexo masculino es el sexo más afectado. También reportaron que las causas externas son: 1) exposición a fuerzas mecánicas inanimadas; 2) caídas; 3) exceso de esfuerzo, viajes y privación; 4) motociclistas lesionados en accidentes de transporte; 5) ocupante de automóvil lesionado en accidente de transporte; 6) contacto con calor y sustancias calientes; 6) agresiones; 7) ocupante de camioneta o furgoneta lesionado en accidente de transporte; 8) exposición a fuerzas mecánicas animadas; 9) ocupante de vehículo de transporte pesado lesionado en accidentes de transporte; y otros ${ }^{3}$.

Asim y cols en el 2006, concluyeron en sus investigaciones que, las condiciones de trabajo, la edad, la seguridad, la experiencia, y el tiempo son factores responsables de accidentes ocupacionales así también, el trabajo temporal o permanente es un generador importante de estos accidentes ${ }^{10}$.

\section{MATERIALES Y MÉTODOS}

Estudio observacional, descriptivo y transversal llevado a cabo en la población trabajadora del área de ventas, compuesta por 206 ayudantes y 81 agentes, de una Cooperativa productora de Refrescos y Bebidas Carbonatadas de la Ciudad de México. Se empleo el Método de Freeman Modificado en la Escuela Nacional de Medicina y Homeopatía del Instituto Politécnico Nacional para fines académicos en el posgrado de Salud Ocupacional, Seguridad e Higiene como metodología para realizar el Diagnóstico Situacional. fueron:

Los pasos seguidos para el desarrollo de las etapas del Método Freeman Modificado

1. Reconocimiento del riesgo.

2. Evaluación de la exposición.

3. Evaluación de la dosis respuesta.

4. Caracterización del riesgo.

\section{RESULTADOS}

Los resultando obtenidos de la aplicación del método Freeman Modificado al proceso de ventas de la empresa refresquera bajo estudio son los siguientes:

Reconocimiento del riesgo: Tras la observación no participativa del proceso de trabajo, se identificaron los puestos implicados y actividades de cada uno de ellos y se delimitaron las etapas del proceso productivo, el cual consta de 9 etapas, representadas en color rojo como muestra el siguiente flujograma. 
Figura 1. Flujograma

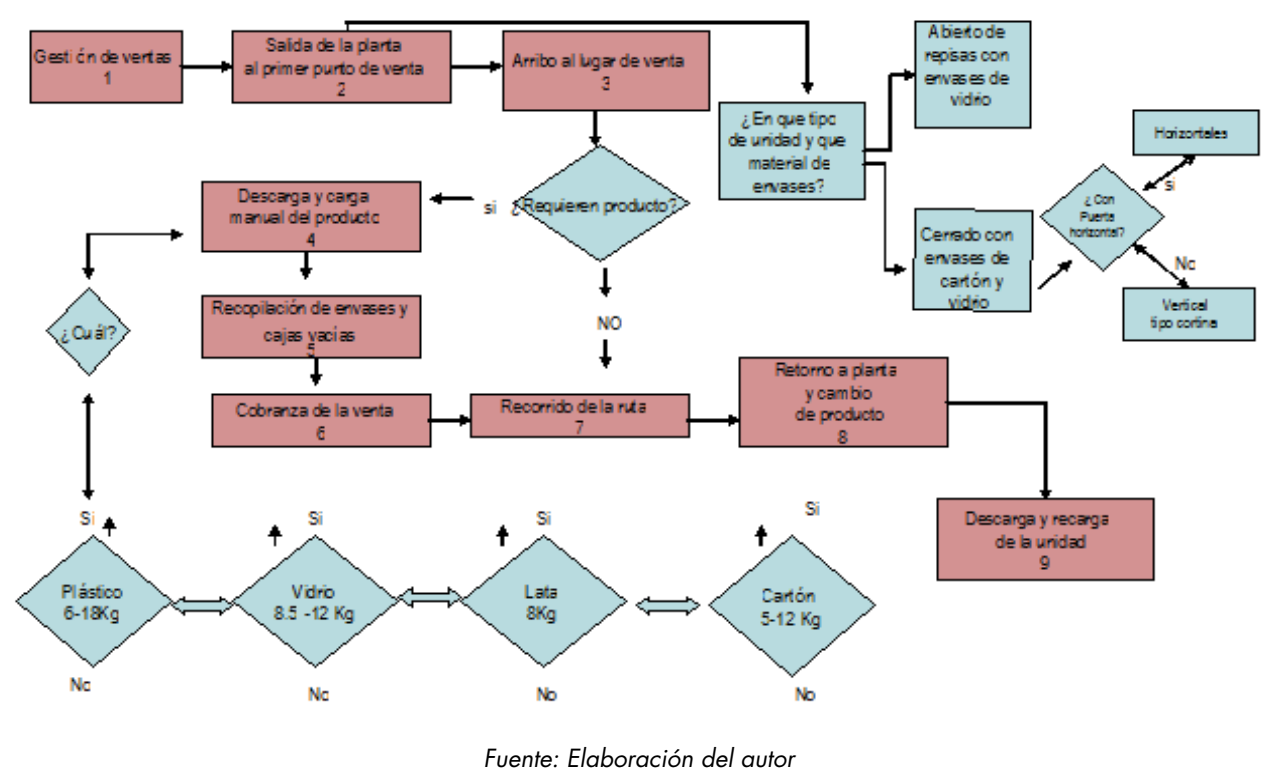

Explicación: Diagrama de las etapas seriadas del proceso de ventas.

Se identificaron 2 puestos de trabajo involucrados en las etapas antes descritas

1. Agente de ventas.

2. Ayudante de ventas.

La tabla IV, muestra los peligros identificados durante toda la observación y análisis de todas las etapas del proceso productivo de ventas.

Tabla IV. Peligros identificados por etapas del proceso productivo del área de ventas

\begin{tabular}{|c|c|}
\hline $\begin{array}{c}\text { Etapas del proceso } \\
\text { productivo de área de } \\
\text { ventas }\end{array}$ & Peligros identificados \\
\hline Etapa 1 & $\begin{array}{l}\text { - Ergonómicos: sobrecarga postural. } \\
\text { - Psicosociales: de tipo organización laboral-relaciones interpersonales conflictivas. } \\
\text { - Físicos: ruido ambiental externo. }\end{array}$ \\
\hline Etapa 2 & $\begin{array}{l}\text { - Ergonómicos: sobrecarga postural, movimientos repetitivos. } \\
\text { - Psicosociales: de tipo organización laboral-ritmo acelerado de la actividad y } \\
\text { jornada mayor a } 8 \text { horas. } \\
\text { - Condición insegura: objetos punzocortantes, traslado inseguro del trabajador, y } \\
\text { tipo de unidad asignada. } \\
\text { - Químicos: contaminación ambiental. } \\
\text { - Físicos: ruido ambiental externo, radiación no ionizante (rayos UV), cambios } \\
\text { térmicos climáticos, vibraciones. }\end{array}$ \\
\hline Etapa 3 & $\begin{array}{l}\text { - Ergonómicos: sobrecarga postural. } \\
\text { - Psicosociales: de tipo organización laboral-relaciones interpersonales conflictivas. } \\
\text { - Biológicos: mordeduras por cánidos. } \\
\text { - Condición insegura: tipo de unidad asignada. }\end{array}$ \\
\hline Etapa 4 & $\begin{array}{l}\text { - Ergonómicos: manejo manual de cargas, movimientos repetitivos, sobrecarga } \\
\text { postura, sobrecarga de trabajo físico. } \\
\text { - Actos inseguros: No emplean el EPP. } \\
\text { - Psicosociales: de tipo organización laboral-ritmo acelerado de la actividad, jornadas } \\
\text { mayores a } 8 \text { horas. } \\
\text { - Condición insegura: falta de capacitación sobre manejo de cargas y uso de EPP. }\end{array}$ \\
\hline
\end{tabular}




\begin{tabular}{|c|c|}
\hline $\begin{array}{c}\text { Etapas del proceso } \\
\text { productivo de área de } \\
\text { ventas }\end{array}$ & Peligros identificados \\
\hline Etapa 5 & $\begin{array}{l}\text { - Ergonómicos: movimientos repetitivos; sobrecarga postural. } \\
\text { - Psicosociales: de tipo organización laboral-jornada mayor a } 8 \text { horas. } \\
\text { - Condición insegura: Objetos punzocortantes. } \\
\text { - Actos inseguros: No emplean guantes. }\end{array}$ \\
\hline Etapa 6 & $\begin{array}{l}\text { - Psicosociales: de tipo organización laboral-relaciones interpersonales conflictivas, } \\
\text { asalto; jornada mayor a } 8 \text { horas, ritmo acelerado de la actividad, sin horario fijo para } \\
\text { alimentos. }\end{array}$ \\
\hline Etapa 7 & $\begin{array}{l}\text { - Ergonómicos: sobrecarga postural manejo manual de cargas. } \\
\text { - Físicos: ruido ambiental externo, radiación no ionizante (rayos UV), cambios } \\
\text { climáticos, vibraciones. } \\
\text { - Psicosociales: de tipo organización laboral-jornada mayor de } 8 \text { horas; sin horario } \\
\text { para alimentos, ritmo acelerado de la actividad. } \\
\text { - Acto inseguro: No emplean guantes. } \\
\text { - Condición insegura: objetos punzocortantes, falta de capacitación sobre el manejo } \\
\text { de cargas y uso de EPP, traslado inseguro del trabajador, tipo de unidad asignada. } \\
\text { - Químicos: contaminación ambiental. } \\
\text { - Biológicos: mordeduras por cánidos. alimentos en la calle. }\end{array}$ \\
\hline Etapa 8 & $\begin{array}{l}\text { - Ergonómicos: sobrecarga postural. } \\
\text { - Psicosociales: de tipo organización laboral-relaciones interpersonales conflictivas, } \\
\text { jornada mayor a } 8 \text { horas. }\end{array}$ \\
\hline Etapa 9 & $\begin{array}{l}\text { - Ergonómicos: sobrecarga postural: manejo manual de cargas, sobrecarga de trabajo } \\
\text { físico. } \\
\text { - Psicosociales: de tipo organización laboral-jornadas mayores a } 8 \text { horas, ritmo } \\
\text { acelerado de la actividad. } \\
\text { - Acto inseguro: no usan guantes. } \\
\text { - Condición insegura: objetos punzocortantes, falta de capacitación sobre el manejo } \\
\text { de cargas y uso del EPP, tipo de unidad asignada. }\end{array}$ \\
\hline
\end{tabular}

Fuente: Elaboración del autor

Con base al análisis del Diagnóstico Situacional elaborado, se focalizó en la identificación únicamente de los factores de riesgo capaces de provocar accidentes de mano y en las etapas del proceso productivo en que se observan, en virtud de que se observó una exposición franca y repetida de la parte distal de los miembros superiores. Estas etapas se resaltan en color amarillo en el mapa de riesgos que a continuación se muestra,

Figura 2. Mapa de Riesgos

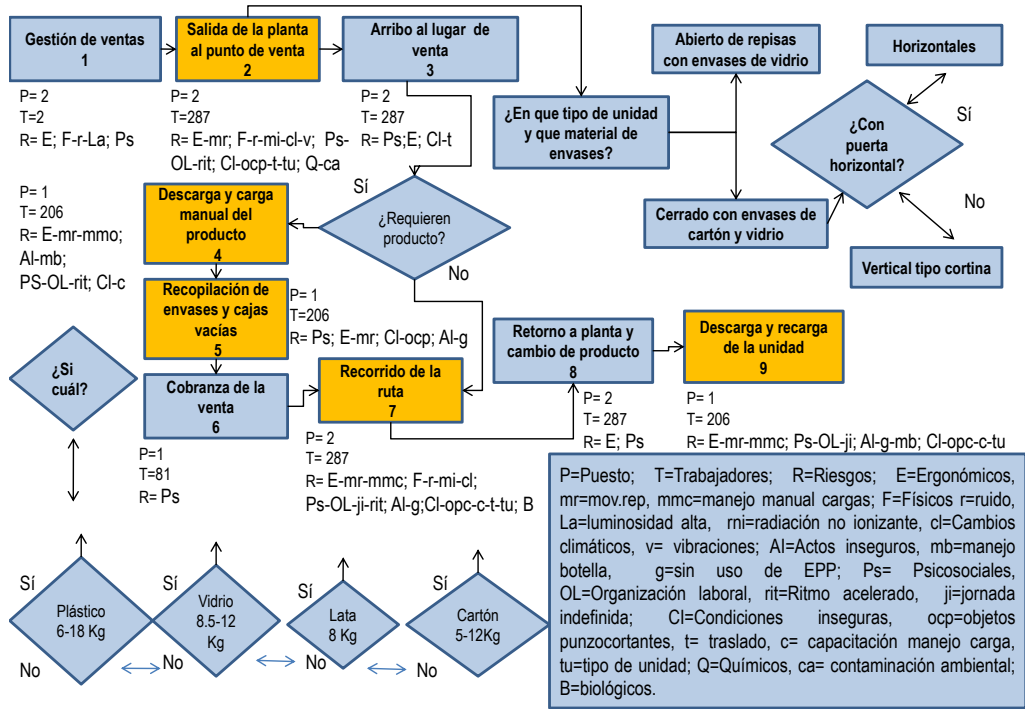

Fuente: Elaboración del autor 
Interpretación: Este mapa de riesgos muestra un PTR (puesto, trabajador y riesgo), que es el número de Puestos implicados y el número de Trabajadores expuestos así como los Riesgos identificados en cada etapa del proceso. Se resaltan en amarillo las etapas en las que se identificaron factores de riesgo relacionados con accidentes de mano. Cabe señalar que esta concatenación de etapas representa sólo a una unidad de trasporte de producto terminado de la empresa.

Evaluación de la exposición: Se evaluaron las variables de frecuencia, duración e intensidad, de cada uno de los peligros o factores de riesgos identificados por etapas del proceso y puestos de trabajo. Cabe señalar que en la intensidad de la exposición, el único factor de riesgo que fue evaluado con un método validado fue la sobrecarga postural (método MAPFRE), en el resto de los peligros no se contaba con datos disponibles por parte de la empresa, pues las mediciones o evaluaciones no las había realizado la organización en ese momento, por lo que en casos puntualizados se tomaron como base registros de pobre calidad, información de fuentes bibliográficas consultadas o de los expertos prácticos.

La evaluación de la exposición permitió observar que los trabajadores expuestos a los diferentes peligros identificados se mantienen en contacto directo con una frecuencia de 6 días a la semana, bajo las mismas condiciones, y con duraciones que van de 1 hora hasta 14 horas por jornada, con concentraciones diversas; también se observó que en la gran mayoría de peligros identificados no se cuenta con registros que midan sus concentraciones, por lo que se estimaron rangos con base en la literatura estudiada.

Evaluación dosis respuesta: En la evaluación dosis respuesta, se encontró que los peligros identificados han tenido impacto en la salud entre los trabajadores del área de ventas, que van desde problemas osteomusculares crónicos hasta lesiones agudas como accidentes, pérdidas de infraestructura, todo esto fue obtenido de escuetos registros internos de la empresa de los años 2004 y 2005. Pese a estar de manifiesto todos estos efectos a la salud en el personal, la organización implemento pocas medidas para el control de los mismos y no existen registros de los resultados obtenidos por la implementación de dichas medidas.

Caracterización del riesgo: Partiendo de la caracterización y jerarquización de los factores de riesgo "generales" identificados en la primera etapa del diagnóstico situacional "Reconocimiento del riesgo", se extrajeron únicamente los factores de riesgo que son capaces de generar los accidentes de mano, respetando la jerarquización ya elaborada.

\section{Factores de Riesgo "Generales"}

1. Físicos: ruido ambiental, vibraciones, radiaciones no ionizantes (rayos U.V).

- Químicos: contaminación ambiental.

- Biológicos: cánidos (mordedura).

- Psicosociales: relaciones interpersonales conflictivas, asalto, ritmo acelerado de trabajo.

- Condiciones Inseguras: traslado del trabajador sobre la defensa trasera del camión.

2. Ergonómicos: sobrecarga postural, movimientos repetitivos, manejo manual de cargas, sobrecarga de trabajo físico.

- Actos inseguros: no uso de equipo de protección personal.

- Condiciones inseguras: falta de capacitación sobre el manejo de cargas, tipo de unidad (por el tipo de puerta que incrementa el riesgo de accidentes).

3. Físicos: cambios climáticos de temperatura.

- Biológicos: ingesta de alimentos vendidos en la calle.

- Psicosociales: jornada mayor a 8 horas (hasta 14 horas), sin horario y lugar fijo para alimentarse.

- Condiciones inseguras: objetos punzocortantes. 
4. Físicos: hiperluminosidad.

- Resultados focalizados y jerarquizados para accidentes de mano, obtenidos del diagnóstico situacional.

\section{Factores de Riesgo de Accidentes de Mano}

Estos son:

1. Psicosociales de organización laboral (ritmo acelerado de la actividad) Condición Insegura (traslado del personal en la defensa trasera del camión).

2. Ergonómicos (movimientos repetitivos, manejo manual de cargas)

- Acto Inseguro (omisión del uso del equipo de protección personal)

- Condición Insegura (tipo de unidad, puerta y material transportado; y falta de capacitación)

3. Psicosociales de organización laboral (jornada indefinida mínima de 8 horas máxima de 14)

- Condición Insegura (objetos punzocortantes en cajas)

La aplicación del método del Diagnóstico Situacional, mostró que las etapas del proceso productivo con mayor riesgo para los accidentes de mano son cinco: la salida de la planta al primer punto de venta; la descarga y carga manual del producto; la etapa de recopilación de envases y cajas vacías; el recorrido de la ruta; y la descarga y recarga de producto de la unidad, al término de la jornada.

Un resultado importante del Diagnóstico Situacional fue la determinación de los factores de riesgo que se asocian con los accidentes de mano, y que fueron: Factores Psicosociales de Organización; Actos y Condiciones Inseguras; y Ergonómicos. Esto, para un mejor estudio y bajo la perspectiva de la Teoría de Sistemas, se reagruparon en tres subsistemas: Ergonómicos, Seguridad, y Capacitación.

\section{CONCLUSIONES}

Los factores de riesgo presentes en la generación de accidentes de mano en el área de ventas de una industria refresquera son: en primer lugar los factores psicosociales debidos al ritmo de la actividad; condición insegura por falta de normatividad en seguridad. En segundo lugar, los factores ergonómicos y actos inseguros por omisión del uso de equipo de protección personal y condiciones inseguras específicamente, falta de capacitación y uso de recursos inseguros proporcionados al trabajador. En tercer lugar, factores psicosociales debido a jornadas mayores de 8 horas y menores de 14 .

Los factores de riesgo mencionados se deben diagnosticar puntualmente y evidenciar en las empresas dedicadas a la producción de bebidas frutales y gasificadas, que buscan disminuir los accidentes de mano a través de medidas preventivas y correctivas.

\section{REFERENCIAS BIBLIOGRÁFICAS}

1. Bohórquez L, compilador. La atención de los riesgos de trabajo como parte de la calidad total [bibliografía]. México: Instituto Mexicano del Seguro Social. Coordinación de Salud en el Trabajo; 1993. 1-17.

2. Ortega V. Costo e Impacto de los Riesgos de Trabajo. [internet]. México; abril 1999 [citado 2007 Enero 20]; Disponible en: www.medspain.com/ant/n4_abr99/costo.html

3. Memorias Estadísticas de Salud en el trabajo [CD-ROM]. México: Instituto Mexicano del Seguro Social. 1999-2009. CD-ROM: 1.

4. Pérez MG. Diseño holístico de un sistema de prevención y control de accidentes de mano, de una empresa refresquera [Tesis de maestría]. [Escuela Nacional de Medicina y Homeopatía]: Instituto Politécnico Nacional; 2009. 250p. 
5. Ortega E. Santillán R. Factores de riesgo, tipo de gravedad y secuelas permanentes en las lesiones de mano de origen profesional en Veracruz. Boletín de Salud en el Trabajo IMSS. 2005; 46(8):1-2.

6. David S, Goel K. Knowledge, Attitude, and Practice of Sugarcane Crusher Towards Hand Injury Prevention Strategies in Indian. Inj Prev. 2001; 7: 329-330.

7. Chow C, Lee H, Lau J. Transiente Risk Factors for Acute Traumatic Hand Injuries: a Case-Crossover Study in Hong Kong. Occup Environ Med. 2007; 64:47-52.

8. Tan K. Manual Materials Handling: a Survey of Risks, and the Selection and Training of Workers in Singapore. Ergonomics. 1987; 30(2):299-304.

9. Guía técnica para la evaluación y prevención de los riesgos relativos a la Manipulación manual de cargas [Internet]. España: INSHT; c1997 [citado 2008 Octubre 02]. Disponible en: http://www2.uca.es/serv/ comite_empresa/salud_laboral/web/gt_manual\%20de\%20cargas.htm

10. Asim S, Anjali N, Pranab K. Occupational Injury Proneness in Indian Women: A Survey in Fish Processing Industries. J Occup Med and Toxicol. 2006; 1(23):1-5.

11. O’Neill R, compilador. Instrucción operativo-ergonomía 01: manipulación manual de cargas [Internet] España: Universidad Politécnica de Valencia. Servicio integrado de prevención y salud laboral; 1999. 16p. cita.

12. Palsson B, Strömberg U, Ohlsson K. Absence Attriibuted to Incapacity and Occupational Disease/ Accidents among Famale and Male Workers in the Fish-Proccessing Industry. Occup. Med. 1998; 48 (5):289-295.

13. Bazroy J, Roy G, Sahai A. Magnitude and Risk Factors of Injuries in a Glass Bottle Manufacturing Plant. J Occup Health. 2003; 45: 53-59

14. Asim S, Kujkarni P, Chaudhuip R, Saiyed H. Occupacional Injuries: Is Job Security a Factor? Indian J Med Sci. 2005; 59(9):375-381

15. Jiménez N, Alvea G. Accidentes de trabajo: Un perfil general. Revista facultad de medicina [internet]. 2005 [citado 2008]; 48(4): Disponible en: www.ejournal.unam.mx/rfm/no48-4/RFM48404.pdf

16. Rodríguez H, González V, Orta F. Reincorporación Laboral en Lesiones de Mano por Accidentes de Trabajo entre Trabajadores IMSS y de Empresas Afiliadas. Boletín de Salud en el Trabajo IMSS. 2006; 51(9):2.

17. Villanueva S, Palacios R, Morales O. Incidencia de accidentes de trabajo en empleados de tortillerías y molinos. Boletín de Salud en el Trabajo IMSS. 2003; 32(6):2.

18. Sousa V, Bouzas J, Albuquerque P. Occupational accidents: social insurance cost and work day lost. Rev Saúde Pública. 2006; 40(6):1-8.

19. Sorock G, Lombardi D, Hauser R. A case-crossover study of transient risk factor for occupational acute hand injury. Occup Environ Med. 2004; 61:305-311.

20. Fabiano B, Curró F, Pastorino R. Occupational injuries in Italy: risk factors and long term trend (1951-98). Occup. Environ. Med.2001; 58:330-338.

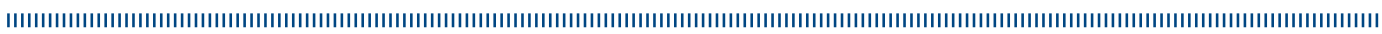

